\title{
Induced Subgraphs of Bounded Degree and Bounded Treewidth ${ }^{\star}$
}

\author{
Prosenjit Bose ${ }^{1}$, Vida Dujmović ${ }^{1}$, and David R. Wood ${ }^{2}$ \\ 1 School of Computer Science \\ Carleton University \\ Ottawa, Canada \\ $\{$ jit,vida\}@scs.carleton.ca \\ 2 Departament de Matemàtica Aplicada II \\ Universitat Politècnica de Catalunya \\ Barcelona, Spain \\ david.wood@upc.edu
}

\begin{abstract}
We prove that for all $0 \leq t \leq k$ and $d \geq 2 k$, every graph $G$ with treewidth at most $k$ has a 'large' induced subgraph $H$, where $H$ has treewidth at most $t$ and every vertex in $H$ has degree at most $d$ in $G$. The order of $H$ depends on $t, k, d$, and the order of $G$. With $t=k$, we obtain large sets of bounded degree vertices. With $t=0$, we obtain large independent sets of bounded degree. In both these cases, our bounds on the order of $H$ are tight. For bounded degree independent sets in trees, we characterise the extremal graphs. Finally, we prove that an interval graph with maximum clique size $k$ has a maximum independent set in which every vertex has degree at most $2 k$.
\end{abstract}

\section{Introduction}

The 'treewidth' of a graph has arisen as an important parameter in the Robertson/Seymour theory of graph minors and in algorithmic complexity. See Bodlaender [2] and Reed [7] for surveys on treewidth. The main result of this paper, proved in Section 5, states that every graph $G$ has a large induced subgraph of bounded treewidth in which every vertex has bounded degree in $G$. The order of the subgraph depends on the treewidth of $G$, the desired treewidth of the subgraph, and the desired degree bound. Moreover, we prove that the bound is best possible in a number of cases.

Before that, in Sections 2 and 3 we consider two relaxations of the main result, firstly without the treewidth constraint, and then without the degree constraint. That is, we determine the minimum number of vertices of bounded degree in a graph of given treewidth (Section 2), and we determine the minimum number of vertices in an induced subgraph of bounded treewidth, taken over all graphs of given treewidth (Section 3). This latter result is the first ingredient

\footnotetext{
* Research of P. Bose and V. Dujmović is supported by NSERC. Research of D. Wood is supported by grant MEC SB2003-0270.
} 
in the proof of the main result. The second ingredient is in Section 4, where we prove that the subgraph of a $k$-tree induced by the vertices of bounded degree has surprisingly small treewidth.

A graph with treewidth 0 has no edges. Thus our results pertain to independent sets for which every vertex has bounded degree in $G$. Here our bounds are tight, and in the case of trees, we characterise the extremal trees. Furthermore, by exploiting some structural properties of interval graphs that are of independent interest, we prove that every interval graph with no $(k+2)$-clique has a maximum independent set in which every vertex has degree at most $2 k$. These results are presented in Section 6.

\subsection{Preliminaries}

Let $G$ be a graph. All graphs considered are finite, undirected, and simple. The vertex-set and edge-set of $G$ are denoted by $V(G)$ and $E(G)$, respectively. The number of vertices of $G$ is denoted by $n=|V(G)|$. The subgraph induced by a set of vertices $S \subseteq V(G)$ has vertex set $S$ and edge set $\{v w \in E(G): v, w \in S\}$, and is denoted by $G[S]$.

A $k$-clique $(k \geq 0)$ is a set of $k$ pairwise adjacent vertices. Let $\omega(G)$ denote the maximum number $k$ such that $G$ has a $k$-clique. A chord of a cycle $C$ is an edge not in $C$ whose endpoints are both in $C$. $G$ is chordal if every cycle on at least four vertices has a chord. The treewidth of $G$ is the minimum number $k$ such that $G$ is a subgraph of a chordal graph $G^{\prime}$ with $\omega\left(G^{\prime}\right) \leq k+1$.

A vertex is simplicial if its neighbourhood is a clique. For each vertex $v \in$ $V(G)$, let $G \backslash v$ denote the subgraph $G[V(G) \backslash\{v\}]$. The family of graphs called $k$-trees $(k \geq 0)$ are defined recursively as follows. A graph $G$ is a $k$-tree if either (a) $G$ is a $(k+1)$-clique, or (b) $G$ has a simplicial vertex $v$ whose neighbourhood is a $k$-clique, and $G \backslash v$ is a $k$-tree.

By definition, the graph obtained from a $k$-tree $G$ by adding a new vertex $v$ adjacent to each vertex of a $k$-clique $C$ is also a $k$-tree, in which case we say $v$ is added onto $C$. For every $k$-tree $G$ on $n$ vertices, $\omega(G)=k+1$; $G$ has minimum degree $k$; and $G$ has $k n-\frac{1}{2} k(k+1)$ edges, and thus $G$ has average degree $2 k-k(k+1) / n$. It is well known that the treewidth of a graph $G$ equals the minimum number $k$ such that $G$ is a spanning subgraph of a $k$-tree.

We will express our results using the following notation. Let $G$ be a graph. Let $V_{d}(G)=\left\{v \in V(G): \operatorname{deg}_{G}(v) \leq d\right\}$ denote the set of vertices of $G$ with degree at most $d$. Let $G_{d}=G\left[V_{d}(G)\right]$. A subset of $V_{d}(G)$ is called a degree- $d$ set. For an integer $t \geq 0$, a $t$-set of $G$ is a set $S$ of vertices of $G$ such that the induced subgraph $G[S]$ has treewidth at most $t$. Let $\alpha^{t}(G)$ be the maximum number of vertices in a $t$-set of $G$. Let $\alpha_{d}^{t}(G)$ be the maximum number of vertices in a degree- $d t$-set of $G$. Observe that $\alpha_{d}^{t}(G)=\alpha^{t}\left(G_{d}\right)$.

Let $\mathcal{G}$ be a family of graphs. Let $\alpha^{t}(\mathcal{G})$ be the minimum of $\alpha^{t}(G)$, and let $\alpha_{d}^{t}(\mathcal{G})$ be the minimum of $\alpha_{d}^{t}(G)$, taken over all $G \in \mathcal{G}$. Let $\mathcal{G}_{n, k}$ be the family of $n$-vertex graphs with treewidth $k$. Note that every graph in $\mathcal{G}_{n, k}$ has at least $k+1$ vertices. These definitions imply the following. Every graph $G \in \mathcal{G}$ has $\alpha_{d}^{t}(G) \geq \alpha_{d}^{t}(\mathcal{G})$ and $\alpha^{t}(G) \geq \alpha^{t}(\mathcal{G})$. Furthermore, there is at least one graph $G$ 
for which $\alpha_{d}^{t}(G)=\alpha_{d}^{t}(\mathcal{G})$, and there is at least one graph $G$ for which $\alpha^{t}(G)=$ $\alpha^{t}(\mathcal{G})$. Thus the lower bounds we derive in this paper are universal and the upper bounds are existential.

As described above, our main result is a lower bound on $\alpha_{d}^{t}\left(\mathcal{G}_{n, k}\right)$ that is tight in many cases. Here, lower and upper bounds are 'tight' if they are equal when ignoring the terms independent of $n$. Many of our upper bound constructions are based on the $k$-th power of an $n$-vertex path $P_{n}^{k}$. This graph has vertex set $\left\{v_{1}, v_{2}, \ldots, v_{n}\right\}$ and edge set $\left\{v_{i} v_{j}:|i-j| \leq k\right\}$. Obviously $P_{n}^{k}$ is a $k$-tree.

For $t=k$, a degree- $d t$-set in a graph $G$ with treewidth $k$ is simply a set of vertices with degree at most $d$. Thus in this case, $\alpha_{d}^{k}(G)=\left|V_{d}(G)\right|$. At the other extreme, a graph has treewidth 0 if and only if it has no edges. A set of vertices $I \subseteq V(G)$ is independent if $G[I]$ has no edges. Thus a 0 -set of $G$ is simply an independent set of vertices of $G$. As is standard, we abbreviate $\alpha^{0}(G)$ by $\alpha(G)$, $\alpha_{d}^{0}(G)$ by $\alpha_{d}(G)$, etc. An independent set $I$ of $G$ is maximum if $|I| \geq|J|$ for every independent set $J$ of $G$. Thus $\alpha(G)$ is the cardinality of a maximum independent set of $G$.

\section{Large Subgraphs of Bounded Degree}

In this section we prove tight lower bounds on the number of vertices of bounded degree in graphs of treewidth $k$. We will use the following result of Bose et al. [3].

Lemma 1 ([3]). Let $G$ be a graph on $n$ vertices, with minimum degree $\delta$, and with average degree $\alpha$. Then for every integer $d \geq \delta$,

$$
\left|V_{d}(G)\right| \geq\left(\frac{d+1-\alpha}{d+1-\delta}\right) n .
$$

Theorem 1. For all integers $k \geq 0$ and $d \geq 2 k-1$,

$$
\lim _{n \rightarrow \infty} \frac{\alpha_{d}^{k}\left(\mathcal{G}_{n, k}\right)}{n}=\frac{d-2 k+1}{d-k+1} .
$$

Proof. First we prove a lower bound on $\alpha_{d}^{k}\left(\mathcal{G}_{n, k}\right)$. Let $G$ be a graph in $\mathcal{G}_{n, k}$ with $\alpha_{d}^{k}(G)=\alpha_{d}^{k}\left(\mathcal{G}_{n, k}\right)$. If a vertex $v$ of $G$ has degree at most $d$ in a spanning supergraph of $G$, then $v$ has degree at most $d$ in $G$. Thus we can assume that $G$ is a $k$-tree. Hence $G$ has minimum degree $k$ and average degree $2 k-k(k+1) / n$. By Lemma 1,

$$
\begin{aligned}
\alpha_{d}^{k}\left(\mathcal{G}_{n, k}\right)=\left|V_{d}(G)\right| & \geq\left(\frac{d+1-2 k+k(k+1) / n}{d+1-k}\right) n \\
& =\left(\frac{d-2 k+1}{d-k+1}\right) n+\frac{k(k+1)}{d-k+1} .
\end{aligned}
$$

Now we prove an upper bound on $\alpha_{d}^{k}\left(\mathcal{G}_{n, k}\right)$ for all $n \equiv 2 k(\bmod d-k+1)$, and for all $k \geq 0$ and $d \geq 2 k-1$. Let $s$ be the integer such that $n-2 k=s(d-k+1)$. 
Then $s \geq 0$. We now construct a graph $G \in \mathcal{G}_{n, k}$. Initially let $G=P_{(s+2) k}^{k}$ be the $k$-th power of the path $\left(v_{1}, v_{2}, \ldots, v_{(s+2) k}\right)$. Let $r=d-2 k+1$. Then $r \geq 0$. Add $r$ vertices onto the clique $\left(v_{i k+1}, v_{i k+2}, \ldots, v_{i k+k}\right)$ for each $1 \leq i \leq s$. Thus $G$ is a $k$-tree, as illustrated in Figure 1. The number of vertices in $G$ is

$$
(s+2) k+s r=(s+2) k+s(d-2 k+1)=s(d-k+1)+2 k=n .
$$

Each vertex $v_{i}, k+1 \leq i \leq(s+1) k$, has degree $2 k+r=d+1$. Hence such a vertex is not in a degree- $d$ set. The remaining vertices all have degree at most $d$. Thus

$$
\alpha_{d}^{k}\left(\mathcal{G}_{n, k}\right) \leq \alpha_{d}^{k}(G)=\left|V_{d}(G)\right|=r s+2 k=\left(\frac{d-2 k+1}{d-k+1}\right) n+\frac{2 k^{2}}{d-k+1}
$$

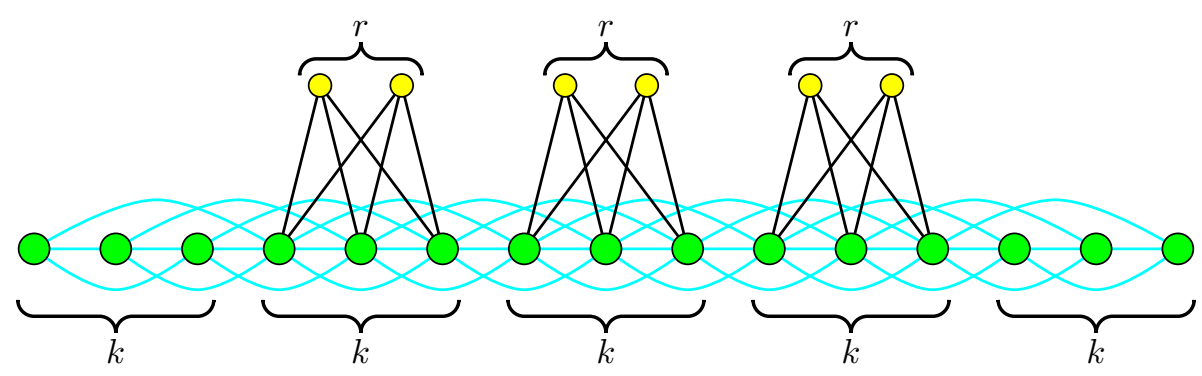

Fig. 1. The graph $G$ with $k=3, d=7$, and $s=3$ (and thus $r=2$ ).

Observe that the difference between the lower and upper bounds in (1) and (3) is only

$$
\frac{2 k^{2}-k(k+1)}{d+1-k}=\frac{k(k-1)}{d+1-k} \leq k-1 .
$$

It is easily seen that for all $\epsilon>0$, there is an $n_{0}$ such that for all $n \geq n_{0}$,

$$
0 \leq \frac{\alpha_{d}^{k}\left(\mathcal{G}_{n, k}\right)}{n}-\frac{d-2 k+1}{d-k+1} \leq \epsilon .
$$

Therefore the sequence $\left\{\alpha_{d}^{k}\left(\mathcal{G}_{n, k}\right) / n: n \geq 2 k\right\}$ converges to $\frac{d-2 k+1}{d-k+1}$.

\section{$3 \quad$ Large Subgraphs of Bounded Treewidth}

We now prove a tight bound on the maximum order of an induced subgraph of bounded treewidth in a graph of treewidth $k$.

Theorem 2. For all integers $n$ and $0 \leq t \leq k$,

$$
\alpha^{t}\left(\mathcal{G}_{n, k}\right)=\left(\frac{t+1}{k+1}\right) n .
$$


Proof. First we prove the lower bound. Let $G$ be a graph in $\mathcal{G}_{n, k}$. First suppose that $G$ is a $k$-tree. By definition, $V(G)$ can be ordered $\left(v_{1}, v_{2}, \ldots, v_{n}\right)$ so that for each vertex $v_{i}$, the predecessors $\left\{v_{j}: j<i, v_{i} v_{j} \in E(G)\right\}$ of $v_{i}$ are a clique of $\min \{k, i-1\}$ vertices. Now colour $G$ greedily in this order. That is, for $i=$ $1,2, \ldots, n$, assign to $v_{i}$ the minimum positive integer (a colour) not already assigned to a neighbour of $v_{i}$. Clearly $k+1$ colours suffice. Let $S$ be the union of the $t+1$ largest colour classes (monochromatic set of vertices). Thus $|S| \geq$ $(t+1) n /(k+1)$. For each vertex $v_{i}$ in $S$, the predecessors of $v_{i}$ that are in $S$ and $v_{i}$ itself form a clique, and thus have pairwise distinct colours. Thus $v_{i}$ has at most $t$ predecessors in $S$, and they form a clique in $G[S]$. Hence $G[S]$ has treewidth at most $t$, and $S$ is the desired $t$-set. Now suppose that $G$ is not a $k$-tree. Then $G$ is a spanning subgraph of a $k$-tree $G^{\prime}$. Thus $G^{\prime}$ has a $t$-set $S$ with at least $(t+1) n /(k+1)$ vertices. Now $G[S]$ is a subgraph of $G^{\prime}[S]$. Thus $G[S]$ also has treewidth at most $t$.

For the upper bound, we now show that every $t$-set of $P_{n}^{k}$ has at most $(t+$ $1) n /(k+1)$ vertices. First suppose that $t=0$. A 0 -set is an independent set. Clearly every independent set of $P_{n}^{k}$ has at most $n /(k+1)$ vertices. Now consider the case of general $t$. Let $S$ be a $t$-set of $P_{n}^{k}$. By the above bound, $P_{n}^{k}[S]$ has an independent set $I$ of at least $|S| /(t+1)$ vertices. Now $I$ is also an independent set of $P_{n}^{k}$. Thus $|I| \leq n /(k+1)$. Hence $|S| /(t+1) \leq n /(k+1)$, and $|S| \leq$ $(t+1) n /(k+1)$.

\section{Structure of Bounded Degree Subgraphs}

In this section we study the structure of the subgraph of a $k$-tree induced by the vertices of bounded degree. We first prove that in a $k$-tree with sufficiently many vertices, not all the vertices of a clique have low degree. A clique $C=\left(v_{1}, v_{2}, \ldots, v_{k}\right)$ of a graph $G$ is said to be ordered by degree if $\operatorname{deg}_{G}\left(v_{i}\right) \leq \operatorname{deg}_{G}\left(v_{i+1}\right)$ for all $1 \leq i \leq k-1$.

Theorem 3. Let $G$ be a $k$-tree on $n \geq 2 k+1$ vertices. Let $\left(u_{1}, u_{2}, \ldots, u_{q}\right)$ be a clique of $G$ ordered by degree. Then $\operatorname{deg}_{G}\left(u_{i}\right) \geq k+i-1$ for all $1 \leq i \leq q$.

Note that Theorem 3 is not true if $n \leq 2 k$, as the statement would imply that a $(k+1)$-clique has a vertex of degree $n$. Thus the difficulty in an inductive prove of Theorem 3 is the base case. Theorem 3 follows from the following stronger result with $n \geq 2 k+1 \geq k+q$.

Lemma 2. Let $G$ be a $k$-tree on $n$ vertices. Let $C=\left(u_{1}, u_{2}, \ldots, u_{q}\right)$ be a clique of $G$ ordered by degree. If $n \geq k+q$ then

$$
\operatorname{deg}_{G}\left(u_{i}\right) \geq k+i-1,1 \leq i \leq q ;
$$

otherwise $n \leq k+q-1$, and

$$
\operatorname{deg}_{G}\left(u_{i}\right) \geq \begin{cases}k+i-1 & \text { if } 1 \leq i \leq n-k-1 \\ n-1 & \text { if } n-k \leq i \leq q .\end{cases}
$$


Proof. We proceed by induction on $n$. In the base case, $G$ is a $(k+1)$-clique, and every vertex has degree $k$. The claim follows trivially. Assume the result holds for $k$-trees on less than $n$ vertices. Let $C$ be a $q$-clique of a $k$-tree $G$ on $n \geq k+2$ vertices. Since every $k$-tree on at least $k+2$ vertices has two nonadjacent simplicial vertices [4], at least one simplicial vertex $v$ is not in $C$. Since $n \geq k+2$ and $v$ is simplicial, the graph $G_{1}=G \backslash v$ is a $k$-tree on $n-1$ vertices. Now $C$ is a $q$-clique of $G_{1}$. Let $C=\left(u_{1}, u_{2}, \ldots, u_{q}\right)$ be ordered by degree in $G_{1}$. By induction, if $n \geq k+q+1$ then

$$
\operatorname{deg}_{G_{1}}\left(u_{i}\right) \geq k+i-1,1 \leq i \leq q ;
$$

otherwise $n \leq k+q$, and

$$
\operatorname{deg}_{G_{1}}\left(u_{i}\right) \geq \begin{cases}k+i-1 & \text { if } 1 \leq i \leq n-k-2 \\ n-2 & \text { if } n-k-1 \leq i \leq q\end{cases}
$$

First suppose that $n \geq k+q+1$. Then by $(6), \operatorname{deg}_{G}\left(u_{i}\right) \geq \operatorname{deg}_{G_{1}}\left(u_{i}\right) \geq k+i-1$, and (4) is satisfied. Otherwise $n \leq k+q$. Let $B=\left\{u_{n-k-1}, u_{n-k}, \ldots, u_{q}\right\}$. Then $|B| \geq 2$, and by (7), every vertex in $B$ has degree $n-2$ in $G_{1}$. That is, each vertex in $B$ is adjacent to every other vertex in $G_{1}$. Let $X$ be the set of neighbours of $v$. Since $v$ is simplicial, $X$ is a $k$-clique. At most one vertex of $B$ is not in $X$, as otherwise $X \cup B$ would be a $(k+2)$-clique of $G_{1}$. Without loss of generality, this exceptional vertex in $B$, if it exists, is $u_{n-k-1}$. The other vertices in $B$ are adjacent to one more vertex, namely $v$, in $G$ than in $G_{1}$. Thus $\operatorname{deg}_{G}\left(u_{i}\right) \geq k+i-1$ for all $1 \leq i \leq n-k-1$, and $\operatorname{deg}_{G}\left(u_{i}\right)=n-1$ for all $n-k \leq i \leq q$. Hence (5) is satisfied.

We can now prove the main result of this section.

Theorem 4. For all integers $1 \leq k \leq \ell \leq 2 k$, and for every $k$-tree $G$ on $n \geq \ell+2$ vertices, the subgraph $G_{\ell}$ of $G$ induced by the vertices of degree at most $\ell$, has treewidth at most $\ell-k$.

Proof. Let $C=\left(u_{1}, u_{2}, \ldots, u_{q}\right)$ be a clique of $G$ ordered by degree. Suppose, for the sake of contradiction, that there are at least $\ell-k+2$ vertices of $C$ with degree at most $\ell$. Let $j=\ell-k+2$. Since $C$ is ordered by degree, $\operatorname{deg}\left(u_{j}\right) \leq \ell$. Since $n \geq \ell+2$, we have $j \leq n-k$. By Lemma $2, \operatorname{deg}\left(u_{j}\right) \geq k+j-1$ (unless $j=n-k$, in which case $\operatorname{deg}\left(u_{j}\right)=n-1 \geq \ell+1$, which is a contradiction). Hence $k+j-1 \leq \ell$. That is, $k+(\ell-k+2)-1 \leq \ell$, a contradiction. Thus $C$ contributes at most $\ell-k+1$ vertices to $G_{\ell}$, and $\omega\left(G_{\ell}\right) \leq \ell-k+1$. Now, $G_{\ell}$ is an induced subgraph of $G$, which is chordal. Thus $G_{\ell}$ is chordal. Since $\omega\left(G_{\ell}\right) \leq \ell-k+1$, $G_{\ell}$ has treewidth at most $\ell-k$.

Note the following regarding Theorem 4 :

- There are graphs of treewidth $k \geq 2$ for which the theorem is not true. For example, for any $p \geq k+1$, consider the graph $G$ consisting of a $(k+1)$ clique $C$ and a $p$-vertex path with one endpoint $v$ in $C$. Then $G$ has at least 
$2 k+1$ vertices, has treewidth $k$, and every vertex of $G$ has degree at most $k$, except for $v$ which has $\operatorname{deg}(v)=k+1$. For $\ell=k, G_{\ell}$ is comprised of two components, one a $k$-clique and the other a path, in which case $G_{\ell}$ has treewidth $k-1>\ell-k=0$. For $k+1 \leq \ell \leq 2 k-1, G_{\ell}=G$ has treewidth $k>\ell-k$.

- The theorem is not true if $k \leq n \leq \ell+1$. For example, for any $1 \leq k \leq$ $\ell \leq 2 k-1$, the $k$-tree obtained by adding $\ell+1-k$ vertices onto an initial $k$-clique has $\ell+1$ vertices, maximum degree $\ell$, and treewidth $k>\ell-k$.

- The case of $\ell=k$ is the well-known fact that in a $k$-tree with at least $k+2$ vertices, distinct simplicial vertices are not adjacent. Put another way, the set of simplicial vertices of a $k$-tree with at least $k+2$ vertices is a 0 -set.

\section{$5 \quad$ Large Subgraphs of Bounded Treewidth and Bounded Degree}

The following theorem is the main result of the paper.

Theorem 5. For all integers $0 \leq t \leq k, d \geq 2 k$, and $n \geq 2 k+1$,

$$
\alpha_{d}^{t}\left(\mathcal{G}_{n, k}\right) \geq\left(\frac{d-2 k+1}{d-\frac{3}{2} k+1+\frac{t(t+1)}{2(k+1)}}\right)\left(\frac{t+1}{k+1}\right) n+\frac{k(t+1)}{d-\frac{3}{2} k+2+\frac{t(t+1)}{2(k+1)}}
$$

Proof. Let $G$ be a graph in $\mathcal{G}_{n, k}$ with $\alpha_{d}^{t}(G)=\alpha_{d}^{t}\left(\mathcal{G}_{n, k}\right)$. A degree- $d$-set of a spanning supergraph of $G$ is a degree- $d t$-set of $G$. Thus we can assume that $G$ is a $k$-tree.

Consider $\ell$ with $k+t \leq \ell \leq 2 k$. By Theorem $4, G_{\ell}$ has treewidth at most $\ell-k$. Since $t \leq \ell-k$, by Theorem 2 ,

$$
\alpha^{t}\left(G_{\ell}\right) \geq\left(\frac{t+1}{\ell-k+1}\right)\left|V_{\ell}(G)\right|
$$

Since $\ell \leq d, \alpha^{t}\left(G_{\ell}\right) \leq \alpha_{d}^{t}(G)$, which implies that

$$
\left|V_{\ell}(G)\right| \leq\left(\frac{\ell-k+1}{t+1}\right) \alpha_{d}^{t}(G) .
$$

Now, $G$ has $k n-\frac{1}{2} k(k+1)$ edges and minimum degree $k$. Let $n_{i}$ be the number of vertices of $G$ with degree exactly $i$. Thus,

$$
\sum_{i \geq k} i \cdot n_{i}=2|E(G)|=2 k n-k(k+1)=-k(k+1)+\sum_{i \geq k} 2 k \cdot n_{i} .
$$

Thus,

$$
\sum_{i \geq 2 k+1}(i-2 k) n_{i}=-k(k+1)+\sum_{i=k}^{2 k-1}(2 k-i) n_{i}=-k(k+1)+\sum_{i=k}^{2 k-1}\left|V_{i}(G)\right|
$$


and

$$
\sum_{i \geq 2 k+1}(i-2 k) n_{i}=-k(k+1)+\sum_{i=k}^{k+t-1}\left|V_{i}(G)\right|+\sum_{i=k+t}^{2 k-1}\left|V_{i}(G)\right| .
$$

By (8),

$$
\begin{aligned}
\sum_{i \geq 2 k+1}(i-2 k) n_{i} & \leq-k(k+1)+t \cdot\left|V_{k+t}(G)\right|+\sum_{i=k+t}^{2 k-1} \frac{(i-k+1) \cdot \alpha_{d}^{t}(G)}{t+1} \\
& \leq-k(k+1)+t \cdot \alpha_{d}^{t}(G)+\frac{\alpha_{d}^{t}(G)}{t+1} \sum_{i=t+1}^{k} i \\
& =-k(k+1)+\alpha_{d}^{t}(G)\left(t+\frac{1}{t+1}\left(\frac{k(k+1)-t(t+1)}{2}\right)\right) \\
& =-k(k+1)+\alpha_{d}^{t}(G)\left(\frac{t(t+1)+k(k+1)}{2(t+1)}\right) .
\end{aligned}
$$

Since $d \geq 2 k$,

$-k(k+1)+\alpha_{d}^{t}(G)\left(\frac{t(t+1)+k(k+1)}{2(t+1)}\right) \geq \sum_{i \geq d+1}(i-2 k) n_{i} \geq(d-2 k+1) \sum_{i \geq d+1} n_{i}$.

Hence,

$$
\left|V_{d}(G)\right|=n-\sum_{i \geq d+1} n_{i} \geq n+\frac{k(k+1)}{d-2 k+1}-\alpha_{d}^{t}(G)\left(\frac{t(t+1)+k(k+1)}{2(t+1)(d-2 k+1)}\right) .
$$

By Theorem 2,

$$
\begin{aligned}
\alpha_{d}^{t}(G) & =\alpha^{t}\left(G_{d}\right) \\
& \geq \frac{t+1}{k+1}\left|V_{d}(G)\right| \\
& \geq \frac{(t+1) n}{k+1}+\frac{k(t+1)}{d-2 k+1}-\alpha_{d}^{t}(G)\left(\frac{t(t+1)+k(k+1)}{2(k+1)(d-2 k+1)}\right) .
\end{aligned}
$$

It follows that

$$
\alpha_{d}^{t}(G) \geq \frac{(d-2 k+1)(t+1) n+k(k+1)(t+1)}{\left(d-\frac{3}{2} k+1\right)(k+1)+\frac{1}{2} t(t+1)} .
$$

The result follows.

A number of notes regarding Theorem 5 are in order:

- Theorem 5 with $t=k$ is equivalent to the lower bound in Theorem 1 . 
- For $d<2 k$, no result like Theorem 5 is possible, since $\alpha_{d}^{t}\left(P_{n}^{k}\right)=2(t+1)$.

- The proof of Theorem 5 is similar to a strategy developed by Biedl and Wilkinson [1] for finding bounded degree independent sets in planar graphs.

We now prove an existential upper bound on the cardinality of a degree- $d$ $t$-set.

Theorem 6. For all integers $k \geq 1$ and $d \geq 2 k-1$ such that $2(d-2 k+1) \equiv 0$ $(\bmod k(k+1))$, there are infinitely many values of $n$, such that for all $0 \leq t<k$,

$\alpha_{d}^{t}\left(\mathcal{G}_{n, k}\right) \leq\left(\frac{d-2 k+1}{d-\frac{3}{2} k+1}\right)\left(\frac{t+1}{k+1}\right) n+\frac{(k-1)(t+1)(d-2 k+1)+k(t+1)(k+1)}{\left(d-\frac{3}{2} k+1\right)(k+1)}$.

Proof. Our construction employs the following operation. Let $G$ be a $k$-tree containing an ordered $k$-clique $C=\left(v_{1}, v_{2}, \ldots, v_{k}\right)$. A block at $C$ consists of $k+1$ new vertices $\left\{x_{1}, x_{2}, \ldots, x_{k+1}\right\}$ where $x_{1}$ is added onto the $k$-clique $\left\{v_{1}, v_{2}, \ldots, v_{k}\right\}$; $x_{2}$ is added onto the $k$-clique $\left\{v_{1}, v_{2}, \ldots, v_{k-1}, x_{1}\right\} ; x_{3}$ is added onto the $k$-clique $\left\{v_{1}, v_{2}, \ldots, v_{k-2}, x_{1}, x_{2}\right\}$; and so on, up to $x_{k+1}$ which is added onto the $k$-clique $\left\{x_{1}, x_{2}, \ldots, x_{k}\right\}$. Clearly the graph obtained by adding a block to a $k$-clique of a $k$-tree is also a $k$-tree

Our graph is parameterised by the positive integer $n_{0} \geq 2 k+3$. Initially let $G$ be the $k$-th power of a path $\left(v_{1}, v_{2}, \ldots, v_{n_{0}}\right)$. Note that any $k+1$ consecutive vertices in the path form a clique. Let $r$ be the non-negative integer such that $2(d-2 k+1)=r k(k+1)$. Add $r$ blocks to $G$ at $\left(v_{i}, v_{i+1}, \ldots, v_{i+k-1}\right)$ for each $3 \leq i \leq n_{0}-k-1$, as illustrated in Figure 2 .

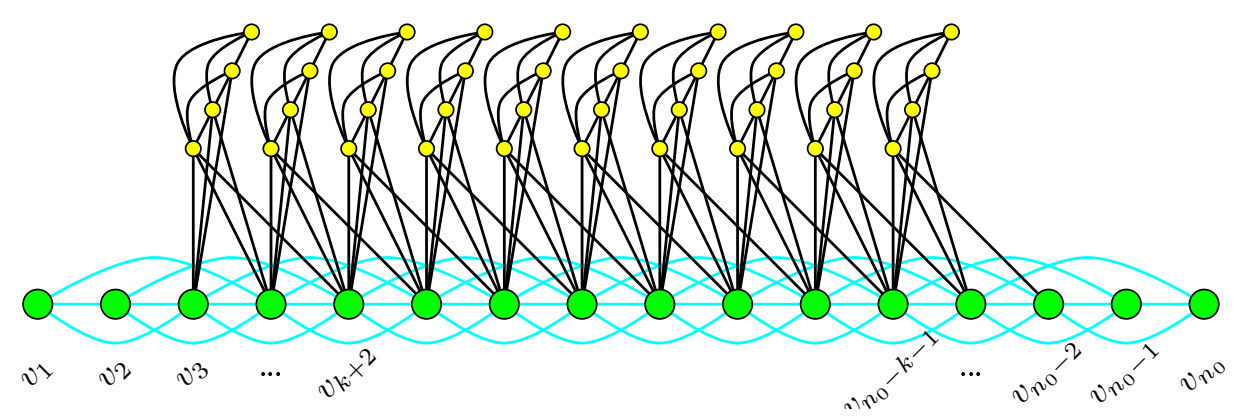

Fig. 2. The graph $G$ with $k=3$ and $d=11$ (and thus $r=1$ ).

$G$ is a $k$-tree with $n=n_{0}+r(k+1)\left(n_{0}-(k+3)\right)$ vertices. Let $S$ be a maximum degree- $d t$-set of $G$. Consider a vertex $v_{i}$ for $k+2 \leq i \leq n_{0}-k-1$. Since $n_{0} \geq 2 k+3$ there is such a vertex. The degree of $v_{i}$ is

$$
2 k+r \sum_{i=1}^{k} i=2 k+\frac{1}{2} r k(k+1)=d+1
$$


Thus $v_{i} \notin S$. Since each block $\left\{x_{1}, x_{2}, \ldots, x_{k+1}\right\}$ is a clique, and treewidth- $t$ graphs have no $(t+2)$-clique, at most $t+1$ vertices from each block are in $S$. Similarly, since $\left\{v_{1}, v_{2}, \ldots, v_{k+1}\right\}$ and $\left\{v_{n_{0}-k}, v_{n_{0}-k+1}, \ldots, v_{n_{0}}\right\}$ are cliques, at most $t+1$ vertices from each of these sets are in $S$. Thus

$$
\alpha_{d}^{t}\left(\mathcal{G}_{n, k}\right) \leq \alpha_{d}^{t}(G)=|S| \leq(t+1)\left(r\left(n_{0}-(k+3)\right)+2\right) .
$$

Substituting the equality $n_{0}=\frac{n+r(k+1)(k+3)}{1+r(k+1)}$ into (9),

$$
\frac{\alpha_{d}^{t}\left(\mathcal{G}_{n, k}\right)}{t+1} \leq \frac{r(n+k-1)+2}{1+r(k+1)} .
$$

The claimed bound on $\alpha_{d}^{t}\left(\mathcal{G}_{n, k}\right)$ follows by substituting the equality $r=\frac{2(d-2 k+1)}{k(k+1)}$ into (10). Observe that $n$ is a function of $n_{0}$ and $n_{0}$ is independent of $d$. Thus there are infinitely many values of $n$ for each value of $d$.

\section{Bounded Degree Independent Sets}

Intuitively, one would expect that a maximum independent set would not have vertices $v$ of high degree, as this would prevent the many neighbours of $v$ from being in the independent set. In this section, we explore the accuracy of this intuition in the case of $k$-trees. Recall that $\alpha_{d}(G)$ is the maximum cardinality of a degree- $d$ independent set in a graph $G$.

Motivated by applications in computational geometry, the previously known results regarding bounded degree independent sets have been for planar graphs $[6,5,8,1]$. The best results were obtained by Biedl and Wilkinson [1], who proved tight bounds (up to an additive constant) on $\alpha_{d}(G)$ for planar $G$ with $d \leq 15$. For $d \geq 16$ there is a gap in the bounds.

Theorem 2 with $t=0$ proves that every $n$-vertex graph $G$ with treewidth $k$ has $\alpha(G) \geq n /(k+1)$, and that this bound is tight for $P_{n}^{k}$. Theorem 5 with $t=0$ gives the following lower bound on the size of a degree- $d$ independent set in a graph of treewidth $k$ (for all $k \geq 1$ and $d \geq 2 k$ ):

$$
\alpha_{d}\left(\mathcal{G}_{n, k}\right) \geq\left(\frac{d-2 k+1}{d-\frac{3}{2} k+1}\right)\left(\frac{n}{k+1}\right)+\frac{k}{d-\frac{3}{2} k+1} .
$$

Note that such a bound is not possible for $d<2 k$ since $\alpha_{d}\left(P_{n}^{k}\right)=2$ for $d<2 k$.

Theorem 6 proves the corresponding upper bound. In particular, for all $k \geq 1$, there are infinitely many values of $d$, and for each such $d$, there are infinitely many values of $n$ for which

$$
\alpha_{d}\left(\mathcal{G}_{n, k}\right) \leq\left(\frac{d-2 k+1}{d-\frac{3}{2} k+1}\right)\left(\frac{n}{k+1}\right)+\frac{(k-1)(d-2 k+1)+k(k+1)}{\left(d-\frac{3}{2} k+1\right)(k+1)} .
$$

These lower and upper bounds are tight. In fact, they differ by at most one. We conclude that

$$
\lim _{n \rightarrow \infty} \lim _{d \rightarrow \infty} \frac{\alpha_{d}\left(\mathcal{G}_{n, k}\right)}{n}=\frac{d-2 k+1}{\left(d-\frac{3}{2} k+1\right)(k+1)}
$$




\subsection{Trees and Interval Graphs}

$\mathcal{G}_{n, 1}$ is precisely the family of $n$-vertex forests. Observe that Theorems 5 and 6 with $k=1$ and $t=0$ prove that for all $d \geq 1$,

$$
\alpha_{d}\left(\mathcal{G}_{n, 1}\right)=\frac{(d-1) n+2}{2 d-1} .
$$

A tree $T$ for which $\alpha_{d}(T)=\frac{(d-1) n+2}{2 d-1}$ is called $\alpha_{d}$-extremal. We omit the proof of the following characterisation of the $\alpha_{d}$-extremal trees. A tree is $d$-regular if every vertex has degree 1 or $d$, and there is at least one vertex of degree $d$.

Theorem 7. Let $d$ be a positive integer. A tree $T$ on $n \geq 5$ vertices is $\alpha_{d^{-}}$ extremal if and only if $T$ is obtained from a $(d+1)$-regular tree by subdividing every leaf-edge once.

A graph $G$ is an interval graph if one can assign to each vertex $v \in V(G)$ a closed interval $\left[L_{v}, R_{v}\right] \subseteq \mathbb{R}$ such that $v w \in E(G)$ if and only if $\left[L_{v}, R_{v}\right] \cap$ $\left[L_{w}, R_{w}\right] \neq \emptyset$. An interval graph $G$ has tree-width equal to $\omega(G)+1$. (In fact, it has path-width equal to $\omega(G)+1$.) Thus the previous results of this paper apply to interval graphs. However, for bounded degree independent sets in interval graphs, we can say much more, as we show in this section. In an interval graph, it is well known that we can assume that the endpoints of the intervals are distinct. We say a vertex $w$ is dominated by a vertex $v$ if $L(v)<L(w)<R(w)<R(v)$.

Lemma 3. Let $G$ be an interval graph with $\omega(G) \leq k+1$. Suppose $G$ has a vertex $v$ with $\operatorname{deg}(v) \geq 2 k+1$. Then there is a vertex $w$ that is dominated by $v$ and $\operatorname{deg}(w) \leq 2 k-1$.

Proof. For each vertex $y \in V(G)$, let $A(y)=\{x \in V(G): L(x)<L(y)<R(x)\}$ and $B(y)=\{x \in V(G): L(x)<R(y)<R(x)\}$. Observe that $x$ is dominated by $y$ if and only if $x y \in E(G)$ but $x \notin A(y) \cup B(y)$. Also $|A(y)| \leq k$ as otherwise $A(y) \cup\{y\}$ would be a clique of at least $k+2$ vertices. Similarly $|B(y)| \leq k$. Thus $|A(y) \cup B(y)| \leq 2 k$.

Now consider the given vertex $v$. Since $\operatorname{deg}(v) \geq 2 k+1, v$ has a neighbour $u \notin A(v) \cup B(v)$. Thus $u$ is dominated by $v$. Let $w$ be a vertex with the shortest interval that is dominated by $v$. That is, if $u$ and $w$ are dominated by $v$, then $R(w)-L(w) \leq R(u)-L(u)$. Thus $w$ does not dominate any vertex, and every neighbour of $w$ is in $A(w) \cup B(w)$. Now $|A(w)| \leq k,|B(w)| \leq k$, and $v \in$ $A(w) \cap B(w)$. Thus $\operatorname{deg}(w) \leq 2 k-1$.

Note that Lemma 3 with $k=1$ is the obvious statement that a vertex of degree at least three in a caterpillar is adjacent to a leaf.

Theorem 8. Every interval graph $G$ with $\omega(G) \leq k+1$ has a degree-2k maximum independent set. That is, $\alpha_{2 k}(G)=\alpha(G)$. 
Proof. Let $I$ be a maximum independent set of $G$. If $I$ contains a vertex $v$ with $\operatorname{deg}(v) \geq 2 k+1$, apply Lemma 3 to obtain a vertex $w$ dominated by $v$ such that $\operatorname{deg}(w) \leq 2 k-1$. Replace $v$ by $w$ in $I$. The obtained set is still independent, since every neighbour of $w$ is also adjacent to $v$, and is thus not in $I$. Apply this step repeatedly until every vertex in $I$ has degree at most $2 k$. Thus $\alpha_{2 k}(G) \geq|I|=\alpha(G)$. By definition, $\alpha_{2 k}(G) \leq \alpha(G)$.

The bound of $2 k$ in Theorem 8 is best possible, since $P_{n}^{k}$ is an interval graph with $\omega(G) \leq k+1$ and only $2 k$ vertices of degree at most $2 k-1$. Thus $\alpha\left(P_{n}^{k}\right)=$ $\lceil n /(k+1)\rceil \gg \alpha_{2 k-1}\left(P_{n}^{k}\right)$.

\section{References}

1. Therese Biedl and Dana F. Wilkinson. Bounded-degree independent sets in planar graphs. Theory of Computing Systems, to appear. In Prosenjit Bose AND Pat Morin, eds., Proc. 13th International Conf. on Algorithms and Computation (ISAAC '02), vol. 2518 of Lecture Notes in Comput. Sci., pp. 416-427. Springer, 2002.

2. HANS L. BodlaENDER. A partial $k$-arboretum of graphs with bounded treewidth. Theoret. Comput. Sci., 209(1-2):1-45, 1998.

3. Prosenjit Bose, Michiel Smid, And David R. Wood. Light edges in degreeconstrained graphs. Discrete Math., 282(1-3):35-41, 2004.

4. Gabriel A. Dirac. On rigid circuit graphs. Abh. Math. Sem. Univ. Hamburg, 25:71-76, 1961.

5. Herbert Edelsbrunner. Algorithms in Combinatorial Geometry. Springer, 1988.

6. DAvid KirkPATRICK. Optimal search in planar subdivisions. SIAM J. Comput., 12(1):28-35, 1983.

7. Bruce A. ReEd. Algorithmic aspects of tree width. In BRuce A. ReEd AND Cláudia L. SAles, eds., Recent Advances in Algorithms and Combinatorics, pp. 85-107. Springer, 2003.

8. Jack SNOEYINK ANd Mard van KReveld. Linear-time reconstruction of Delaunay triangulations with applications. In RAINer E. Burkhard AND GERHARD J. Woeginger, eds., Proc. 5th Annual European Symp. on Algorithms (ESA '97), vol. 1284 of Lecture Notes in Comput. Sci., pp. 459-471. Springer, 1997. 